\title{
Wearable Rehabilitation Device for the Training of Movements Based on the Mapping of the Real Time Angle Values
}

\author{
Pranmya Ratnaparkhi
}

Student, VIT University Bhopal, India

\begin{abstract}
Most of The movement disabilities occur either because of some kind of injury or because of some kind of disorders in the body. Disabilities related to hand movements are more common. These kinds of disabilities require specific training that is to be done in order to regain the normal movements of the specific part. The movements of arms are monitored by measuring the 3D angles made using IMU gyro sensors interfaced with the microcontroller. Mapping of these values is used to generate a specific output which varies with the varying angular values and is used to specify the range of the movements which are required for the training. The magnitude of actuation varies with the varying values of the measured angle which indicate the improvement in the movements. The device is able to set the range of the motion for the movement. The intensity of the buzzer decreases with the increasing value of angle and totally disappears as the pre-set value of the angle is achieved. The basic principle behind the device is the decrease of analog output of the microcontroller calibrated with the value of gyro sensor such that the output decreases with increase in the value of the gyro sensor. This device can be used to monitor the improvement in the movement and also can be used to monitor the accomplishment of the task suggested for movement rehabilitation. As the output of the device is very easy to interpret so can be used by any person. The patient can monitor their movements by just observing changes in the output.
\end{abstract}

Keywords: Movement disabilities, IMU gyro sensor, microcontroller, analog output, pre-set, 3D angles.

\section{INTRODUCTION}

Movement is an important characteristic of living beings and it is one of the important physiological processes which play a vital role in the survival of the living organism. In the case of human beings sometimes this movement is restricted because of various kinds of disabilities that may occur because of many reasons. This kind of disability creates a lot of problems, so it becomes highly important to identify them and treat them as soon as possible to regain normal behavior.
Among all kinds of movement disabilities most of them are associated with that of limbs and as limbs play a very important role so it becomes very important to treat them. This kind of movement can occur because of many reasons. Some disabilities arise because of some kind of disorder in the physiological systems of the person. There are many kinds of disorders that cause this type of movement disabilities. This can be either due to genetic defects or sometime can occur because of some kind of physiological imbalance in the later part of the life. In most of the cases these kinds of disorders are either neural disorders or neuromuscular disorders. This is either because of improper or defective production of some specific molecule or sometimes because of neuro degeneration which leads to this kind of disorders. Sometimes these kinds of disabilities are because of genetic defects like in case of perry syndrome in which certain mutations in certain specific genes cause trouble in normal functioning of the system.

So these are the some disabilities arising due to disorders in the system but most of the movements' disabilities arise because of the physical reasons like injuries which either damages or injures the internal muscles, tendons, ligaments or sometimes bones which results in the movement disability. These injuries may occur because of many physical reasons like accidents or sometimes improper movements which are sometimes beyond the capacity of the normal systems of the body and hence results in either partial damage or sometimes total damage. These tissues like muscles, tendons etc. are responsible for carrying out the movements and require proper care and treatment to recover properly and regain their abilities. There are many kinds of surgical treatments being used to treat these kinds of movement disabilities but apart from this specific training is required in order to regain the normal movements. This kind of training is given by various physiotherapists who help the tissue to recover slowly with the ability to move and again back to their original work.

This training also ensures the growth of the tissues in proper shape and direction to avoid any kind of abnormal growth. This training helps the patients to move their injured parts slowly and continually improve their movement under the observation of experts. This training is done step by step 
according to the progress of the patients. This training also monitors the progress at regular intervals of the time in order to know the recovery of the damaged tissue. This kind of therapy sometimes becomes very tedious for both patients and the experts so in this modern world with emerging technologies there are many assistive wearable devices being developed to assist the patient to regularly train their movements and also monitor their progress. But most of these devices are again very complex to use and also are bulky and very costly so everyone can't afford them. So the system that we designed solves this as it is highly portable, efficient and also very easy to use as it does not require specific expert supervision in order to use it.

The main issue resolved is output in the form of sound so that the output can be easily interpreted and understood and the patient's progress can be monitored. The device is able to track the hand movements and generate output accordingly which is used to analyse the patient's progress. The device is so easy to use and highly interactive. The device measures the real time angular values using gyro sensors and then sets the threshold that a patient's needs to achieve slowly, the device maps these values and generates the analog output and this output indicates to the patient about the movement and whether the threshold set has been reached or not. The threshold can be adjusted manually by the patients depending upon the use.

\section{MATERIALS}

\subsection{ESP32}

ESP32 is a kind of system on chip microcontroller based development board. This board has wifi connectivity along with the dual mode bluetooth connectivity which makes it highly versatile for the use in the IOT based projects. This board uses a tensilica xtensa 1x6 32 bit microprocessor with operating frequency of $160 \mathrm{MHz}$ or $240 \mathrm{MHz}$ and is available both in dual core as well as single core variation. This board has built in systems like various kinds of filters, low noise amplifiers, power management modules and much more. Espress if systems designed this esp32 board which is considered as successor of the esp 8266 board. This board works on the voltage range of around $2.2 \mathrm{~V}$ to $3.6 \mathrm{~V}$. It takes power from either the usb port or from the vin pin it has in its peripherals. The esp32 board has $520 \mathrm{~KB}$ of SRAM and 448 $\mathrm{KB}$ of ROM. Also it has flash size of around $4 \mathrm{Mb}$ which retains the data even if the power is off hence it plays a vital role in the systems where the data needs to be retained. The wifi used in this system is $802.11 \mathrm{~b} / \mathrm{g} / \mathrm{n}$.

The device depending upon the mode of operation can act both as station or access points or sometimes both. It has both modes of bluetooth connectivity which can be used accordingly. Apart from wireless connectivity it is capable of transmitting and receiving data using various data transmission protocols like SPI, I2C, CAN bus 2.0, I2S and UART. It has $4 \times$ SPI, $2 \times$ I2C, $2 \times$ I2S and $3 \times$ UART pins. It has total 34 programmable pins with different modes. The digital I/O pins it has are capable of taking inputs and generating output. It has $2 \mathrm{DAC}$ with 8 bit resolution. Also it has 18 channels of SAR ADC with 12 bit resolution. These SAR ADC channels have special capability of converting the analog waveform to discrete waveform using a binary search in all possible quantization levels before the final convergence of the results. Along with these pins there are also some pins which are touch enabled and are capable of sensing a touch. These pins are based upon capacitive sensing.

The esp32 board has an inbuilt Hall Effect sensor capable of sensing the variations in the magnetic field. It has special channels for infrared remote controllers. The programmable pins are also able to generate the PWM output as per the requirement. Most of the esp32 boards have inbuilt USB to TTL converter chip which is used for the programming of the device and is capable of exchanging the data between the device and the host via usb cable. Some versions of the esp32 board require external USB to TTL converter chips for programming and data exchange.

An external reset button is present which resets the device and along with it a boot button is also present which enables the programming of the device. Esp32 boards can be programmed by using various different platforms and in different languages. Most commonly esp32 boards with arduino cores are programmed using arduino IDE as the arduino IDE has support for the esp32 boards which makes it easier for the user to easily programm the board. The arduino IDE uses a subpart of c++ to program the boards and also has various inbuilt libraries for esp32 boards. The esp32 board is also programmed using micropython which are a subpart of python. Various platforms like Thonny IDE are used to program the esp32 board using micropython.

For programming using micropython some special firmware should be uploaded before the implementation of the codes. Some other platforms like espressino, Mongoose OS, Lua, Espressif mesh development framework, mruby etc are also used for the programming of the esp32 boards. These programming platforms use different programming languages. So Esp32 development boards are highly versatile in nature with wireless connectivity which is an enhancing feature for any kind of development board because of which these boards find greater to use in the field of internet of things and various other developmental purposes. 
ISSN (online): 2581-3048

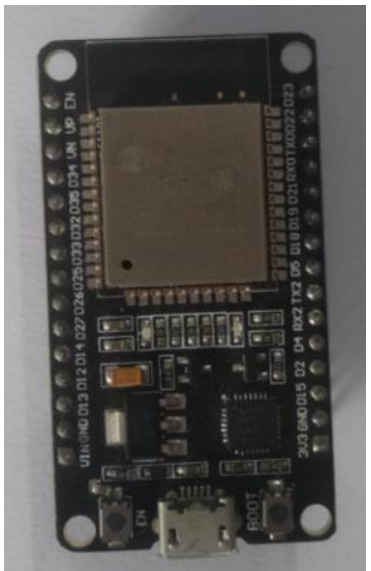

Figure 1: ESP32

\subsection{MPU6050}

These sensors are used to monitor the angular velocity and the orientation of the object in 3D space. This sensor generates electrical signals depending upon the orientation of the object and its acceleration and velocity. Basically mpu6050 is a MEMS based sensor which primarily monitors the acceleration. This is used to analyse the orientation of the object in the $3 \mathrm{~d}$ space using mathematical calculations. The sensor generates electrical signals of different magnitude which are measured by the microcontroller unit which measures and calculates the acceleration. The sensor is capable of measuring orientation, velocity, acceleration etc. This sensor is a MEMS based sensor in which an oscillating spring mass system is created on the micro scale which when oscillates generates different output. The device works in two different parts. First one is the measurement of the acceleration of the object. These measurements are done on the basis of the change in capacitance that arises due to displacement in the micro spring mass system created in the silicon wafer.

The spring mass system starts oscillating as soon as force is applied because of which the small plates of the capacitors move apart which of which the capacitance changes which is monitored by measuring the change in the electrical output. The second part is the working of the gyroscope. This is based on the Coriolis Effect according to which the perpendicular displacement because of the effect of the coriolis force is directly related to the angular rate applied. Now in the sensor module a MEMS system is created on the micro scale in which 4 proof masses are connected together by a spring. When the sensor is rotated the coriolis force starts acting on the MEMS because of which the directions of the proof mass changes. It again has three modes depending upon the axis on which the angular motion is applied. These three modes are Roll, Yaw and Pitch. Now again the different modes cause change in capacitance which are sensed by the sensing unit.
This sensor module works on the voltages ranging from $3 \mathrm{~V}$ to 5V. The sensor module has 6 different pins i.e Vin, Gnd, SCL, SDA, XCL, XDA, INT. SDA and SCL pins are serial data and serial clock pins respectively. These $\mathrm{p}$ [ins are responsible for the transmission of the day from the sensor to the microcontroller unit via $\mathrm{I} 2 \mathrm{C}$ protocol. Interrupt pin is used to check whether the data has been received or not.

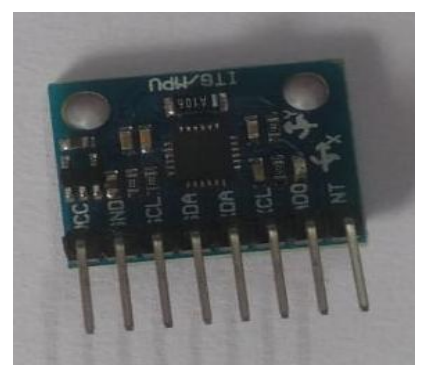

Figure 2: MPU6050

\subsection{Passive buzzer}

Passive buzzer is a device which generates output in the form of sound. This device converts electrical energy into sound energy. Basically it contains a piezo sensitive material which tests oscillation as soon as it receives electrical signals. In case of passive buzzers varying $\mathrm{AC}$ signals are required for the working of the buzzer. The passive buzzers change the intensity of sound produced by them in accordance with the magnitude of the input AC signals applied upon them. The varying $\mathrm{AC}$ signals result in a difference in the oscillation frequency of the piezo resistive material hence sound with different intensities is produced.

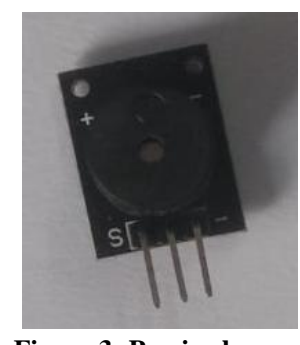

Figure 3: Passive buzzer

\subsection{DPDT Switch}

DPDT switches are used to shift the position of the switch because of which the state change occurs. These switches are used where two different states with different inputs are required so as to perform a specific function.

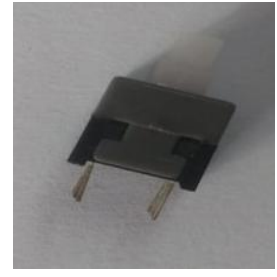

Figure 4: DPDT Switch 
ISSN (online): 2581-3048

\subsection{Arduino IDE}

Arduino IDE is an open source platform provided by the arduino community for the programming and debugging of various arduino development boards and the boards with the arduino support in them. This IDE provides a visual interface between the microcontrollers and the user in order to program the microcontroller as the user. The IDE has many inbuilt boards with the support while some of the other boards packages can be downloaded. Also the IDE provides many custom made and inbuilt libraries for the interfacing with different types of sensors and the actuators. The IDE allows the user to write the code and has an inbuilt compiler for checking the errors. The code can be implemented to the boards through type ports. Also the IDE enables serial communication with the device. This serial data can be visualized either using a serial monitor or serial plotter.

\section{WORKING}

The working of the device is based on the measurement done by the sensor module which is processed by the microcontroller and the output generated is given to the buzzer. This whole process is divided into three steps i.e sensing, processing and output generation.

\subsection{Sensing}

The MPU6050 sensor module is fixed in such a position that it is able to monitor the movements of the limbs. The data form sensor is recorded by the esp32 board. The sensor takes power from the esp32 board itself. As the MPU6050 sensor module uses the I2C data transfer protocol it is interfaced with the esp 32 board using $\mathrm{I} 2 \mathrm{C}$ ports of the board and programmed using the specified libraries. Along with this some specific libraries are used for making the proper calculations and converting the raw data into the readable form. The MPU6050 sensor is able to measure the acceleration and the orientation. The sensor gives data into various formats which are converted according to the use. For analyzing the hand movements the gyro readings are taken into consideration. The sensor first measures the initial position of the limbs and the data is stored by the esp 32 board for further calculations. The sensor module continuously records the angular data and sends it to the esp32 board. As the limbs are moved in 3D space the sensor orientation changes which is analyzed by the esp32 board. The sensor is able to monitor the arm movements in all the three modes i.e roll (along $\mathrm{X}$-axis), pitch(along Y-axis), and yaw(along Z-axis). As the hand moves its angles in the $3 \mathrm{D}$ space changes which play an important role in analyzing the movements. The system also has a DPDT switch. The state of this switch is analyzed by the esp32 board as the state of the switch has an important role in specifying the range of the motion. This switch is used to decide the type and the range that should be used for the movement. The patient in initial training is not allowed to move the arms normally, instead of that there are different levels specified which should be followed by the patients. The DPDT switch allows the patient to opt for initial and final training as per the requirement. The DPDT switch sets the range for the patient, if the patient is in the initial stage the range of the movement would be less and would go on increasing as the initial ranges are completed by the patients. The first state sets the partial range of the movement. As the patient recovers the state can be changed which will set the range to its peak values.

\subsection{Processing}

The data read from the MPU6050 sensor module is read and processed by the esp32 board. The sensor angular values initially twice once initially which is constant throughout the measurement and the second value is variable and changes with orientation. The angle values are mapped using the map function in arduino IDE. The map function in the IDE takes the input range and output range and converts the variable value into the value between the output ranges mentioned in the map function. Expansion of map ():

long $\operatorname{map}($ long $\mathrm{x}$, long in_min, long in_max, long out_min, long out_max)

\{

return $(\mathrm{x}$ - in_min $) *$ (out_max - out_min) / (in_max in_min) + out_min;

\}

In the above expansion $\mathrm{x}$ is the variable that needs to be mapped. The in_min and the in_max are the input ranges of the variable i.e the value in this range should be mapped. The out_min and out_max are the minimum and maximum values of the range in which the variable should be mapped. Using the map() function the angels in range -180 to 180 are converted into values between range 0 to 255 as the PWM output of the esp32 is in this range. This is done once in the setup () function to get the initial value and in loop () function to continuously map the $\mathrm{x}$ angel values into the specified range. The variable generated after mapping of angle value is further mapped to specify the range of the motion. The mapping is done as:

$\mathrm{Xi}=$ initial angel;

$\mathrm{X}=$ angel read by the sensor;

$\mathrm{X} 2=\operatorname{map}(\mathrm{X}, 180,180,0,255)$;

The state of the DPDT switch decides the maximum range upto which the variable should be mapped into desired range. If the stare of the DPDT is LOW then the calibrated value which ensures the partial movement of the arms, is 
ISSN (online): 2581-3048

added to the initial value and if the switch is in HIGH state the calibrated value which ensures the full movement is added to the initial value. The initial angel value and the value set by the DPDT are the minimum and the maximum values between which each value should be mapped. For ex if initial reading of the gyro sensor is 70 so if the state is low then 45 is added into the initial value, and then the angel value between 70 and 115 will be mapped from 255 to 0; Double mapping of the angel value is done to get the maximum PWM value i.e 255 at the initial position and 0 at the peak position up to which the patient should move their hand. Now the final mapping is:

$\mathrm{Xf}=\mathrm{Xi}+45$

$\mathrm{X} 3=\operatorname{map}(\mathrm{X} 2, \mathrm{Xi}, \mathrm{Xf}, 255,0)$;

The main aim of this kind of processing is to set the output range according to the angel values which shows maximum at initial angel value and minimum at the value that the patient need to move the hand up to. This output is used by the passive buzzer.

\subsection{Output}

The data received after the processing is given to the buzzer. The passive buzzer is given the PWM data generated after the processing step. The buzzer receives the maximum value i.e 255 at the initial position and goes on decreasing as the sensory approaches to the present value and becomes zero at the preset position and the sound of the buzzer vanishes. Also the sound intensity of the buzzer goes on decreasing as the movement approaches to the desired value. The values are mapped in such a way that the sound intensity of the buzzer goes on decreasing instead of increasing the intensity because if the intensity is maximum at the present opposition it becomes difficult for the patient to identify the correct position because the intensity of the sound will remain at its high even the position has been reached but when the intensity goes on decreasing it is very easy to identify a position at which he sound vanished.

\section{RESULTS}

The device is able to record and analyse the motion of the limbs and is able to convert it into the desired value. The DPDT switch is able to set the values for the mapping purpose. The sensor continually records the angel values and the values are mapped in order to generate the output as per the requirement. The values are processed such that the output is at its maximum value at the initial position and goes on decreasing as the arm approaches the preset value that the patient needs to achieve. As the output from the esp32 decreases the sound intensity of the passive buzzer also decreases and vanishes completely at the final position. The intensity of the sound is maximum at the initial position and zero at the final position. The device helps the patient to identify the movement and also to track the motion w.r.t to the set values by analysing the output in the form of the sound as it is very to understand the varying sound intensity with the varying values according to the preset values.

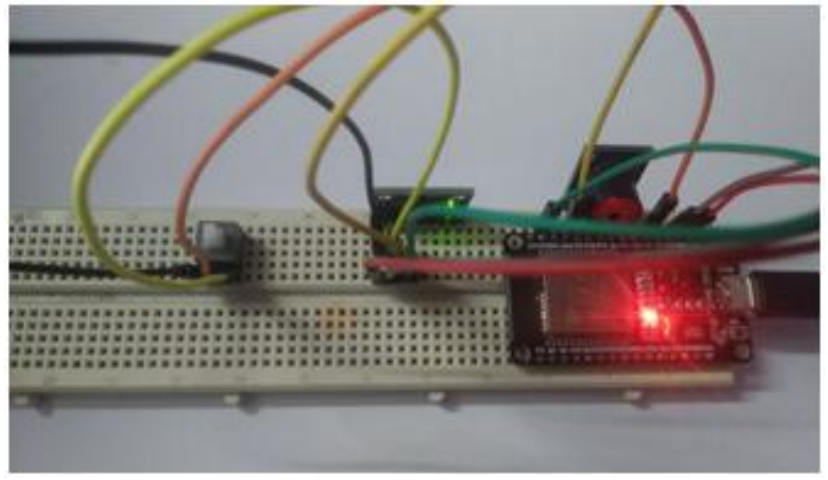

Figure 5: Result

\section{CONCLUSION}

The device is based on the variation of the sound intensity with the variation in the angel values recorded by the MPU6050 sensor module. It is comparatively easy to just understand the changing sound intensity instead of understanding complex mathematical data which is not possible for everyone. This device can help the patients recovering from the movement disabilities by helping them to set the prior movements and angle whether the movement is proper or not. Also the device is very simple to operate as one needs to just set he mode on the DPDT switch as per the requirement and moved the hand upto the position where the sound vanishes, by this the patient can know that he reached the position upto which he has to move his/her arms. As the device uses the sound as the output so it is highly interactive in nature and easily understandable. The patients can train their movements just by themselves.

\section{REFERENCES}

[1] NASAM - National Stroke Association of Malaysia. http://www.nasam.org/english/preventionwhat_is_a_stroke.php, 2013.

[2] Maciejasz, P., Eschweiler, J., Gerlach-Hahn, K., Jansen-Troy, A., Leonhardt, S. A survey on robotic device for upper limb. Journal of Neuro Engineering, 2014.

[3] Chang, W.H., Kim, Y. Robot-assisted therapy in stroke rehabilitation. Journal of Stroke. 2013. p. 174181.

[4] Krebs, H.I., Ferraro, M., Buerger, S.P., Newbery, M.J., Makiyama, A., Sandmann, M., Hogan, N. Rehabilitation robotics: Pilot trial of a spatial 
ISSN (online): 2581-3048

extension for MIT-Manus. Journal of Neuro Engineering.

[5] Lu. C.E. Development of an Upper Limb Robotic Device for Stroke Rehabilitation.

[6] Masiero, S., Armani, M. Rosati, G. Upper-limb robot-assisted therapy in rehabilitation of acute stroke patients: Focused review and results of new randomized controlled trial. Journal of Rehabilitation Research and Development. 2011. p. 355-366.

[7] Kahn, L.E., Zygman, M.L., Rymer, W.Z., Reinkensmeyer, D.J. Robot-assisted reaching exercise promotes arm movement recovery in chronic hemiparetic stroke: A randomized controlled pilot study. 2006. Journal of NeuroEngineering and Rehabilitation.

[8] Rahman, T., Sample, W., Jayakumar, S., King, M.M., Wee, J.Y., Seliktar, R., Alexander, M., Scavina, M., Clark, A. Passive exoskeletons for assisting limb movement. Journal of Rehabilitation Research \& Development. 2006. p. 583-590.

[9] Mihelj, M., Nef, T., Riener, R. ARMin - Towards a six DoF upper limb rehabilitation robot. 2006. Biomedical Robotics and Biomechatronics. 2006. p. 1154-1159.

[10] Annisa, J., Mohamaddan, S., Jamaludin, M.S., Noor Aliah Abd., M., Omar, A., Helmy, H., Norafizah, A. Development of Upper Limb Rehabilitation Robot Prototype for Home Setting.

[11] R. Ambar, M. S. Ahmad, and M. M. Abdul Jamil, "Design and Development of Arm Rehabilitation Monitoring Device", IFMBE proceedings: vol. 35, pp. 781-784, 5th Kuala Lumpur International Conference on Biomedical Engineering (Biomed), Kuala Lumpur, Malaysia, in conjunction with the 8th asian Pacific Conference on Medical and Biological Engineering (APCMBE 2011) 20-23 June 2011, Springer-Verlag Berlin.

[12] Georg Ogris, Matthias Kreil, Paul Lukowicz. "Using Fsr based muscule activity monitoring to recognize manipulative arm gestures", Wearable Computers, 2007. 11th IEEE International Symposium on Wearable Computers, 2007. pp 45-48.

[13] John Sarik, Ionannis Kymissis (2010). Lab Kits Using the Arduino Prototyping Platform. 40th ASEE/IEEE Frontiers in Education Conference, 2010.

[14] Ryan Fitzhenry (2009). Design and Develop Virtual Reality Games Utilising the "Anti-gravity" Arm Support for Stroke Rehabilitation Therapy. Degree thesis 2009, Faculty of Engineering and Surveying, University of Southern Queensland, Australia.
[15] Muscular Dystrophy Association (MDA) ALS Division (2010). Everyday Life with ALS: A Practical Guide. MDA Publications Department.

[16] Peggy A. Houglum. (2010). Therapeutic Exercise for Musculoskeletal Injuries. Human Kinetics Publisher, 229.

[17] Taborri, J.; Palermo, E.; Rossi, S.; Cappa, P. Gait Partitioning Methods: A Systematic Review. Sensors 2016, 16, 66 .

[18] Chiraka Phaisarn, N. Measurement and Analysis System of the Knee Joint Motion in Gait Evaluation for Rehabilitation Medicine. In Proceedings of the Fourth International Conference on Digital Information and Communication Technology and Its Applications (DICTAP), Bangkok, Thailand, 6-8 May 2014.

[19] Moufawad el Achkar, C.; Lenoble-Hoskovec, C.; Paraschiv-Ionescu, A.; Major, K.; Büla, C.; Aminian, K. Physical Behavior in Older Persons during Daily Life: Insights from Instrumented Shoes. Sensors 2016, 16, 1225. Appl. Sci. 2018, 8, 203214 of 14.

[20] Ladha, C.; O’Sullivan, J.; Belshaw, Z.; Asher, L. Gait Keeper: A System for Measuring Canine Gait. Sensors 2017, 17, 309.

[21] Duong, P.D.; Suh, Y.S. Foot Pose Estimation Using an Inertial Sensor Unit and Two Distance Sensors. Sensors 2015, 15, 15888-15902.

[22] Boussaad, M.; Sijobert, B.; Mombaur, K.; Azevedo Coste, C. Robust Foot Clearance Estimation Based on the Integration of Foot-Mounted IMU Acceleration Data. Sensors 2016, 16, 12.

[23] Zhou, Q.; Zhang, H.; Lari, Z.; Liu, Z.; El-Sheimy, N. Design and Implementation of Foot-Mounted Inertial Sensor Based Wearable Electronic Device for Game Play Application. Sensors 2016, 16, 1752.

[24] Mitschke, C.; Heß, T.; Milani, T.L. Which Method Detects Foot Strike in Rearfoot and Forefoot Runners Accurately when Using an Inertial Measurement Unit? Appl. Sci. 2017, 7, 959.

[25] Kok,M.;Hol,J.D.;Schön,T.B.And Optimization-based approach human body motion capture using inertial sensors. IFAC Proc. Vol. 2014, 47, 79-85. 


\section{Citation of this Article:}

Pranmya Ratnaparkhi, "Wearable Rehabilitation Device for the Training of Movements Based on the Mapping of the Real Time Angle Values" Published in International Research Journal of Innovations in Engineering and Technology - IRJIET, Volume 5, Issue 6, pp 35-41, June 2021. Article DOI https://doi.org/10.47001/IRJIET/2021.506008 